Article

\title{
The Relationships between Hip and Knee Extensor Cross-Sectional Area, Strength, Power, and Potentiation Characteristics
}

\author{
Timothy J. Suchomel ${ }^{1, *}$ and Michael H. Stone ${ }^{2}$ \\ 1 Department of Human Movement Sciences, Carroll University, Waukesha, WI 53186, USA \\ 2 Department of Exercise and Sport Sciences, Center of Excellence for Sport Science and Coach Education, \\ East Tennessee State University, Johnson City, TN 37614, USA; stonem@etsu.edu \\ * Correspondence: tsuchome@carrollu.edu; Tel.: +1-262-524-7441
}

Received: 26 February 2017; Accepted: 29 August 2017; Published: 5 September 2017

\begin{abstract}
The purpose of this study was to examine the relationships between muscle cross-sectional area (CSA), maximal strength, power output, and maximum potentiation characteristics. The vastus lateralis and biceps femoris CSA, one repetition maximum (1RM) back squat, 1RM concentric-only half-squat (COHS) strength, static jump power output, and maximum potentiation characteristics of 17 resistance-trained men was assessed during several testing sessions. Pearson's correlation coefficients were used to examine the relationships between CSA, strength, power output, and maximum potentiation measures. Moderate-to-strong relationships existed between CSA and strength measures $(r=0.462-0.643)$ as well as power output $(r=0.396-0.683)$. In addition, moderate-to-strong relationships existed between strength and power output $(r=0.407-0.548)$, while trivial relationships existed between strength and maximum potentiation $(r=-0.013-0.149)$. Finally, small negative relationships existed between CSA and maximum potentiation measures $(r=-0.229-0.239)$. The results of the current study provide evidence of the interplay between muscle CSA, strength, power, and potentiation. Vastus lateralis and biceps femoris CSA may positively influence an individual's back squat and COHS maximal strength and squat jump peak power; however, muscle CSA and absolute strength measures may not contribute to an individual's potentiation capacity. Practitioners may consider implementing resistance training strategies that improve vastus lateralis and biceps femoris size in order to benefit back squat and COHS strength. Furthermore, implementing squatting variations—both full and partial—may benefit jumping performance.
\end{abstract}

Keywords: postactivation potentiation; back squat; half-squat; squat jump; ultrasound; vastus lateralis; biceps femoris

\section{Introduction}

A sequenced progression of training phases can result in the enhanced development of fitness characteristics that contribute to an individual's overall performance. Previous literature has indicated that increases in work capacity and the cross-sectional area (CSA) of the involved musculature during an initial strength-endurance phase may enhance an individual's ability to increase their muscular strength during subsequent training phases [1-3]. Furthermore, increases in an individual's force production capacity and rate of force development during maximal strength training phases may then lead to an increased ability to demonstrate greater power output [4,5], as well as additional abilities such as postactivation potentiation (PAP) [4,6-8]. Additional research supports this notion as muscle CSA was shown to be a strong predictor of the variance in muscular strength and neuromuscular performance (maximal vertical jump performance) in adult men and women [9]. Thus, it may be 
argued that muscle CSA serves as an important foundation on which both muscular strength and power may be built.

A recent review reported strong relationships between measures of muscular strength and a number of performance characteristics including rate of force development, power output, general sport skills (jumping, sprinting, and change of direction), specific sport tasks, and PAP [4]. Although an abundance of literature has examined relationships between back squat strength and performance, a paucity of literature has examined the relationships between squats performed from different depths and performance. Given that muscle activation [10-12] and relative muscular effort [13] may vary with squat depth, it is important to examine these relationships to determine how muscular strength in various positions affects performance. Furthermore, considering that hip and knee extensors are frequently trained during resistance training programs that include squatting variations, it is important to understand how the characteristics of these muscles (e.g., CSA) contribute to muscular strength during these movements. From a practical standpoint, these analyses would provide further information regarding the possible implementation of partial squats into strength training programs as well as provide insight into the relationships between hip and knee extensor size and maximal squat and partial squat strength.

Results of previous studies indicate that participants with greater lower extremity absolute [14-16] and/or relative strength [17-19] may produce greater magnitudes of PAP compared to their weaker counterparts. Additional research demonstrated that one may achieve greater potentiation after increasing their strength [20]. While it appears that muscular strength is a strong predictor of PAP, it is possible that an individual's muscle CSA may also be a predictor of PAP, given its influence on muscular strength. A recent study by Seitz and colleagues [21] indicated that quadriceps CSA was strongly correlated $(r=0.68)$ with maximal voluntary PAP response during isokinetic knee extensions. Although the previous study provides some indication of the relationships between muscle CSA and $\mathrm{PAP}$, it should be noted that the vast majority of potentiation literature has sought to enhance the performance of more complex tasks such as vertical jumping. Thus, it appears that further research is warranted to provide sport scientists and practitioners with information on how muscle CSA relates to maximal PAP during a multi-joint movement. These findings may have practical applications given the attention that potentiation complexes have received within the strength and conditioning literature. Therefore, the purpose of this study was to examine the relationships between muscle CSA, maximal strength, power output, and maximum PAP. Based on the extant literature, it was hypothesized that strong relationships would exist between muscle CSA, strength, and power output and that small relationships would exist between CSA and maximum PAP.

\section{Materials and Methods}

\subsection{Participants}

Seventeen resistance-trained males (age $=24.2 \pm 4.2$ years, height $=180.1 \pm 8.6 \mathrm{~cm}$, body mass $(\mathrm{BM})=86.5 \pm 9.2 \mathrm{~kg}$, one repetition maximum $(1 \mathrm{RM})$ back squat $=164.7 \pm 29.9 \mathrm{~kg}, 1 \mathrm{RM}$ concentric-only half-squat $(\mathrm{COHS})=194.6 \pm 28.2 \mathrm{~kg}$, relative back squat $1 \mathrm{RM}=1.9 \pm 0.3 \mathrm{~kg} / \mathrm{kg} \mathrm{BM}$, relative COHS $1 \mathrm{RM}=2.3 \pm 0.3 \mathrm{~kg} / \mathrm{kg} \mathrm{BM}$ ) who regularly trained with the back squat exercise volunteered to participate in this study. All participants reported that they were performing $\geq$ three resistance sessions per week. Prior to testing, all participants read and signed a written informed consent form. This study was approved by the University's Institutional Review Board.

\subsection{RM Back Squat Testing Session}

The purpose of the 1RM back squat testing session was to establish each participant's 1RM back squat and determine the starting position for the 1RM COHS testing session. Prior to testing, each participant performed a standardized general warm-up (two minutes of stationary cycling at $50 \mathrm{~W}$ at approximately $70 \mathrm{rpm}$ ). This was followed by a dynamic warm-up that included dynamic stretches 
covering a distance of $10 \mathrm{~m}$ (e.g., forward walking lunge, straight leg march, etc.) and five repetitions each of slow bodyweight squats and fast bodyweight squats. Two minutes of rest were provided following the warm-up before the participant started the 1RM back squat test protocol as outlined by previous research [22]. Briefly, each participant performed 5, 5, 3, and 1 warm-up repetition(s) at 30, 50,70 , and $90 \%$ of their self-determined 1RM, respectively. Following the warm-up back squat sets, participants completed maximal back squat attempts, with four minutes of recovery between attempts, at progressively increasing loads until a failed attempt occurred. The loads were determined by the primary investigator and research assistants based on the previous $1 \mathrm{RM}$ attempt. A minimum $2.5 \mathrm{~kg}$ increase was required and all participants achieved their 1RM back squat in four attempts or fewer. All back squat repetitions were performed to a depth where the participant's hip crease dropped below their patella.

After the completion of the 1RM back squat, participants were provided with a self-selected rest period. Following the rest period, each participant squatted with a $20 \mathrm{~kg}$ barbell to a knee angle of $90^{\circ}$ in order to determine the safety bar height for the 1RM COHS that would be performed during the following 1RM COHS session. The knee angle was verified through the use of a manual goniometer and the safety bar heights were adjusted accordingly. After the safety bars were adjusted, the participant positioned themself under the barbell to confirm that their position for the COHS 1RM test was correct.

\subsection{RM Concentric-Only Half-Squat Testing Session}

Each participant returned for the 1RM COHS testing session one week following the 1RM back squat session. The 1RM COHS testing session was used to determine the loads that would be used during the potentiation testing session, and to familiarize the participants with the testing conditions. Prior to testing, participants performed the same warm-up protocol as described above. Similar to the 1RM back squat testing session, the participant began performing warm-up COHS repetitions following a two minute rest period. Warm-up sets of 5, 5, 3, and 1 repetition(s) were performed at $30,50,70$, and $90 \%$ of the participant's estimated 1RM COHS as described by previous research [22]. The 1RM COHS warm-up loads were based on previous pilot testing that indicated that the 1RM COHS was approximately $1.2 \times$ the participant's $1 \mathrm{RM}$ back squat. Following the final warm-up set, participants performed maximal COHS attempts, with four minutes of recovery between attempts, at progressively increasing loads until a failed attempt occurred. All COHS repetitions were performed with the barbell resting on the safety pins of the squat rack with the participant starting with a $90^{\circ}$ knee angle. The participants then used a concentric-only muscle action to finish each repetition [22]. Each participant's 1RM COHS was determined in four attempts or fewer.

\subsection{Potentiation Testing Session}

One week following the 1RM COHS session, participants arrived for the potentiation session. Participants completed the same general warm-up procedures described above before receiving final instructions before they completed their baseline static jumps (SJ) on the force plates. Following warm-up SJs at $50 \%$ and $75 \%$ of their perceived maximum effort, participants performed two maximal effort SJs with one minute of rest between jumps. Participants then began the potentiation protocol two minutes following their baseline jumps. The potentiation protocol was based on previous research $[17,22,23]$ and consisted of five COHS repetitions at $30 \%$, three repetitions at $50 \%$, three repetitions at $70 \%$, and two repetitions at $90 \% 1 \mathrm{RM}$ of the participant's previously established 1RM COHS. All COHS were performed in a ballistic manner as previously described $[17,22,23]$. Two minutes of rest were provided following the sets at 30\% and 50\% $1 \mathrm{RM}$ and four minutes were provided following the set at $70 \% 1 \mathrm{RM}$. Immediately following the final COHS repetition, each participant walked out of the squat rack and stepped onto the force plates. The participant was then instructed to squat down to the "ready position" (i.e., $90^{\circ}$ knee angle) and received a countdown (i.e., "3, 2, 1, jump!"). The participants then performed a SJ using a concentric-only movement to jump 
as high as possible while holding a near weightless $(<1 \mathrm{~kg})$ PVC pipe on their upper back, similar to a high bar back squat position. Subsequent SJs were performed in the same manner every minute up to 10 min following the completion of the potentiation protocol, similar to previous research [17].

\subsection{Ultrasonography}

Prior to performing the warm-up and 1RM back squat protocol, CSA measurements of the participants' right vastus lateralis (VL) and biceps femoris (BF) muscles were assessed using a linear probe scanning head with a 3.4-10.8 MHz bandwidth range (LOGIQ P6, GE Healthcare, Wauwatosa, WI, USA). The probe was coated with a water soluble transmission gel (Aquasonic 100 ultrasound transmission gel, Parker Laboratories, Inc., Fairfield, NJ, USA) and positioned on the surface of the skin to provide acoustic contact without depressing the dermal layer to collect an image. The CSA images for the VL and BF were obtained using a sweep of the muscle in the extended field of view mode with the gain set to $50 \mathrm{~dB}$ and an image depth to $5 \mathrm{~cm}$. For the VL CSA measurements, participants laid on an athletic training table on their left side with their legs together and relaxed with $15^{\circ}$ of knee flexion as measured by a manual goniometer [24]. For the BF CSA measurements, participants laid in a prone position with their feet hanging off the end of the athletic training table. The anatomical location for all CSA measurements was standardized for all participants. VL CSA was measured at $50 \%$ of the distance between the greater trochanter and the lateral condyle of the tibia. BF CSA was measured at $50 \%$ of the distance between the ischial tuberosity and the posterior aspect of the fibular head.

\subsection{Data and Statistical Analyses}

All SJ repetitions were performed on a dual force plate setup (two separate $45.5 \mathrm{~cm} \times 91 \mathrm{~cm}$ force plates; RoughDeck HP, Rice Lake, WI, USA) sampling at $1000 \mathrm{~Hz}$. The SJ data were collected and analyzed using a customized LabVIEW program (2012 Version, National Instruments Co., Austin, TX, USA). Voltage data obtained from the force plates were filtered using a digital low-pass Butterworth filter with a cutoff frequency of $10 \mathrm{~Hz}$ in order to minimize noise within the signal [23]. Static jump peak power (PP) was calculated as the product of force and velocity and as the greatest instantaneous value from the raw power-time data. The average values of PP were calculated between the two baseline repetitions and compared with the values obtained during the SJs performed at each post-stimulus rest interval (i.e., immediately and 1-10 $\mathrm{min}$ ) during the potentiation session. The percentage improvement (i.e., potentiation) in group SJ PP was determined at each rest interval and the greatest mean improvement was used for the correlation analysis. Baseline SJs were used for the reliability analysis. Three CSA measurements were taken for both the VL and BF. Each trial was used for the reliability analysis and the average CSA was used for further statistical analysis.

Two-way random effects mixed model intraclass correlation coefficients (ICC) and typical error (TE) expressed as a coefficient of variation percentage were used to assess inter-trial reliability. A paired-samples $t$-test was used to examine the differences between baseline SJ PP and the time interval in which the maximum PP potentiation occurred. In addition to $95 \%$ confidence intervals (CI), Cohen's d effect sizes were used to indicate the practical significance between baseline PP and maximum PP potentiation. Pearson's correlation coefficients $(r)$ were used to examine the relationships between VL and BF CSA, 1RM back squat, 1RM COHS, SJ PP, and maximum percent PP potentiation. Based on the current sample size, it was determined that a correlation of 0.48 was needed to demonstrate a statistically significant relationship. Effect size and correlation magnitudes were interpreted based on previous scales displayed by Hopkins [25]. All statistical analyses were performed using SPSS 23 (IBM, Armonk, NY, USA) and statistical significance was set at $p \leq 0.05$.

\section{Results}

The inter-trial reliability ICC for VL and BF CSA were both 0.99 and the typical error percentages were $2.5 \%$ and $2.9 \%$, respectively. In addition, the ICC and typical error percentage for SJ PP were 0.98 and $2.0 \%$, respectively. The descriptive data are displayed in Table 1. 
Table 1. Descriptive muscle CSA, strength, power, and maximum potentiation data.

\begin{tabular}{|c|c|c|c|c|c|c|}
\hline Characteristic & $\begin{array}{l}\text { VL CSA } \\
\left(\mathrm{mm}^{2}\right)\end{array}$ & $\begin{array}{c}\text { BF CSA } \\
\left(\mathrm{mm}^{2}\right)\end{array}$ & $\begin{array}{c}\text { 1RM BS } \\
\text { (kg) }\end{array}$ & $\begin{array}{c}\text { 1RM COHS } \\
(\mathrm{kg})\end{array}$ & SJ PP (W) & Max PAP (\%) \\
\hline Mean \pm SD & $32.5 \pm 5.8$ & $18.1 \pm 3.5$ & $164.7 \pm 29.9$ & $195.0 \pm 28.2$ & $4867.2 \pm 638.4$ & $3.9 \pm 3.6$ \\
\hline
\end{tabular}

\subsection{Static Jump Potentiation}

Peak PAP for the participants occurred at two minutes following the potentiation stimulus. Statistically significant differences for SJ PP existed between baseline and PP at two minutes post-stimulus $(t=4.233, p=0.001, \mathrm{~d}=0.29, \mathrm{CI}=90.6-272.2)$. Figure 1 displays the change in $\mathrm{SJ}$ $\mathrm{PP}$ at each time point with Cohen's $\mathrm{d}$ effect sizes indicating the difference in performance compared to baseline.

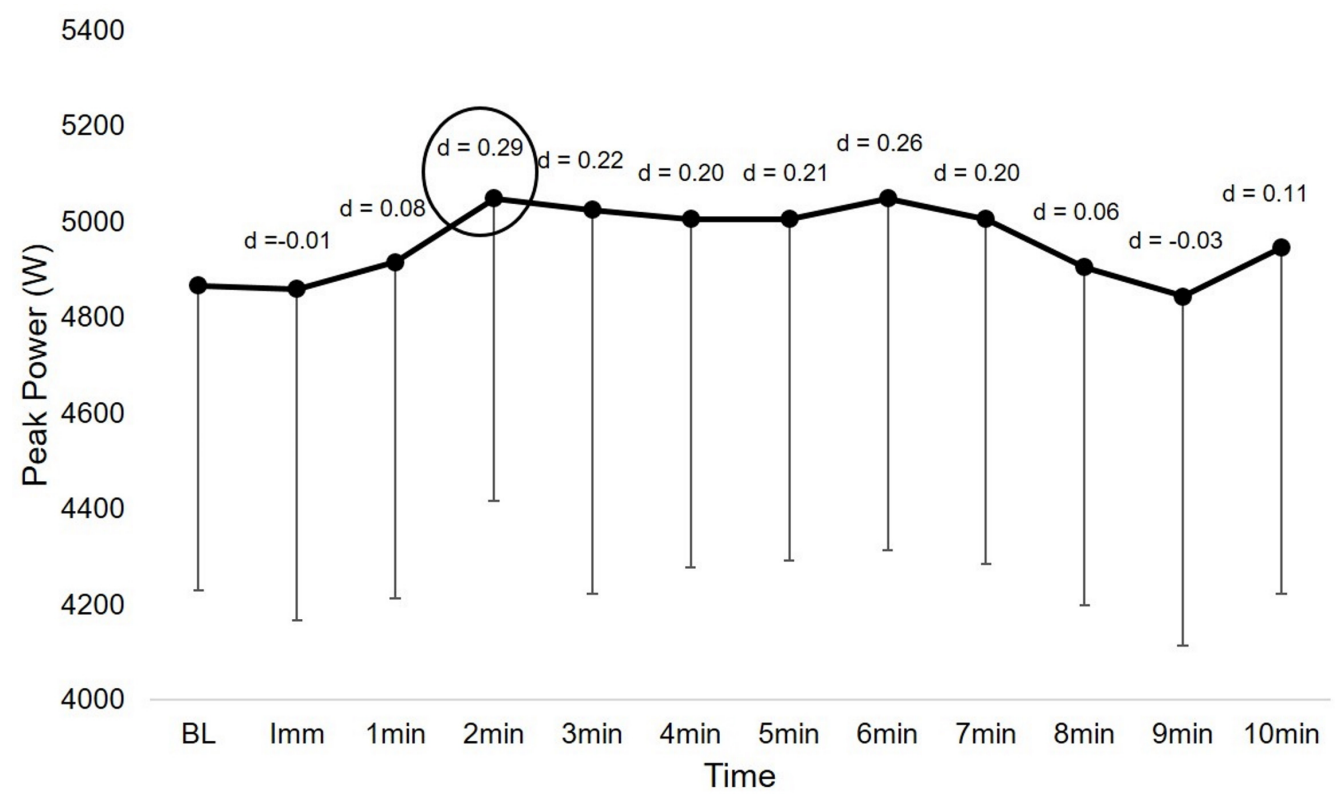

Figure 1. Temporal profile of peak power potentiation with Cohen's d effect size differences from baseline (BL). Notes: Imm = immediately (i.e., $\sim 15 \mathrm{~s}$ following potentiation stimulus); circle $=$ rest interval that demonstrated the greatest potentiation, used for statistical comparison with baseline performance.

\subsection{CSA, Strength, Power, and Potentiation Relationships}

Large positive relationships were present between VL and BF CSA measurements and strength measurements (i.e., 1RM back squat and 1RM COHS), with the exception of a moderate relationship existing between BF CSA and 1RM COHS. In contrast, small negative relationships existed between muscle CSA measurements and maximum potentiation. Large and moderate positive relationships existed between 1RM back squat and 1RM COHS with SJ PP, respectively. In contrast, trivial relationships existed between both strength measures and maximum potentiation; however, follow-up analyses between relative back squat and COHS 1RM and maximum potentiation displayed larger relationships ( $r=0.196$ and $r=0.398$, respectively). Finally, a small negative relationship existed between SJ PP and maximum potentiation. The relationships between muscle CSA, strength, power, and maximum potentiation measures are displayed in Table 2. 
Table 2. Relationships between muscle CSA, strength, power, and maximum potentiation.

\begin{tabular}{ccccccc}
\hline Characteristic & VL CSA & BF CSA & 1RM BS & 1RM COHS & SJ PP & Max PAP \\
\hline VL CSA & 1.000 & & & & & \\
BF CSA & $0.669^{*}$ & 1.000 & & & & \\
1RM BS & $0.643^{*}$ & $0.643^{*}$ & 1.000 & & & \\
1RM COHS & $0.625^{*}$ & 0.462 & $0.897^{*}$ & 1.000 & & \\
SJ PP & 0.396 & $0.683^{*}$ & $0.548^{*}$ & 0.407 & 1.000 & \\
Max PAP & -0.229 & -0.239 & -0.013 & 0.149 & -0.297 & 1.000
\end{tabular}

Notes: $\mathrm{VL}=$ vastus lateralis; $\mathrm{BF}=$ biceps femoris; $\mathrm{CSA}=$ cross-sectional area; $1 \mathrm{RM}=$ one repetition maximum; $\mathrm{BS}=$ back squat; $\mathrm{COHS}=$ concentric-only half-squat; $\mathrm{SJ}=$ static jump; $\mathrm{PP}$ = peak power output; Max PAP = maximum potentiation; ${ }^{*}=$ statistically significant relationship $(p \leq 0.05)$.

\section{Discussion}

The primary findings regarding the relationships with CSA measurements are as follows: first, moderate-large relationships existed between VL and BF CSA and maximal strength measures; second, moderate-large relationships existed between VL and BF CSA measures and SJ PP; third, trivial relationships existed between strength and maximum PAP measures; and finally, small negative relationships existed between VL and BF CSA measures and maximum PAP.

Classic work from Stone et al. [26], Minetti [1], and Zamparo et al. [2] suggests that increasing muscle CSA, particularly in a task specific manner, can lead to an increased ability to improve the muscle's maximal force production capacity (i.e., strength) in the same or similar tasks. From a physiological perspective, an increase in muscle CSA leads to an improved capacity to produce force due to the addition of newly formed sarcomeres. The addition of sarcomeres then increases the number of potential interactions between actin and myosin within the sarcomere (i.e., cross-bridges), causing an increase in the potential force that may be produced by a given muscle. This idea is supported by previous research that displayed increases in muscle fiber pennation angles with increases in muscle hypertrophy [27]. Our results support the previous literature as moderate-to-large relationships existed between the participants' VL and BF CSA and multiple measures of maximal strength. Thus, it appears that a sequenced progression of training phases may allow an earlier phase of training to lay the foundation to potentiate or enhance the subsequent phase(s) of training [3]. However, it should be noted that the constraints of each maximal strength test may have affected the magnitude of some of the relationships. For example, both the VL and BF CSA measures displayed similar large relationships with the participants' back squat strength. In contrast, while a large relationship was displayed between VL CSA and COHS strength, only a moderate relationship was displayed with BF CSA. Although not measured in the current study, previous literature indicated that greater squat depths increased the relative muscular effort of both hip and knee extensors [13]. Thus, it is possible that the relative effort of the BF may have been reduced during the COHS, due to a shallower squat, which may have contributed to a smaller relationship compared to that of the VL.

Muscle CSA measures displayed moderate-to-large relationships with SJ PP, which is similar to previous research $[28,29]$. It is interesting to note that the relationship between the BF CSA and SJ PP produced a stronger correlation compared to VL CSA and SJ PP. However, these differences may be explained by the fiber arrangement of each muscle. Although not measured in the current study, previous research indicated that the hamstring muscles possess a greater number of sarcomeres in series compared to the quadriceps muscles [30]. Based on this fiber arrangement and the length of the muscles, the hamstrings may result in a 30\% greater shortening velocity compared to the quadricep muscles [30], which may ultimately result in greater magnitudes of power output. In contrast, the quadriceps may produce approximately $40 \%$ greater muscle tension compared to the hamstrings [30], likely the result of a larger number of sarcomeres in parallel. The findings of the current study provide further support that muscle CSA may serve as a foundation for both muscle strength and power characteristics.

While moderate-to-strong relationships existed between muscle CSA, strength, and power output, the same cannot be said for the relationships between muscle CSA and maximum PAP. Small negative 
relationships were displayed between both VL and BF CSA and maximum PAP within the current study. Our results are in contrast to previous literature that displayed a large positive relationship $(r=0.68)$ between quadriceps CSA and maximal voluntary PAP response during isokinetic knee extensions [21]. Although conflicting evidence, the complexity of the potentiation task, in this case a SJ, must be taken into consideration. Previous literature discussed how both the characteristics of the participant as well as the potentiation complex may affect whether or not PAP is displayed for vertical jumps [6]. The results of the current study indicate that although a small magnitude of PAP was displayed, larger muscle size (i.e., CSA) may not have been a positive contributing factor. It is probable that other characteristics, such as the nervous system or participant's fiber type, contributed to an enhanced performance.

The current study displayed trivial relationships between strength measures and maximum PAP. These findings are in contrast with previous literature that noted small-to-large relationships between absolute muscular strength and PAP [14-16]. However, it should be noted that larger relationship magnitudes have been displayed between relative strength and PAP [17-19]. As indicated by our follow-up analysis, the relationship magnitudes between relative back squat and COHS strength and maximum potentiation grew to small-to-moderate magnitudes. Thus, while the primary analysis of the current study required the use of absolute strength rather than relative strength units (i.e., relative to kilogram of body mass), it is possible that the latter may contribute to a greater extent to PAP.

As mentioned in the previous paragraph, a potential limitation of the current study was the use of absolute strength and power measurements instead of relative values when assessing the relationships between variables. However, it should be noted that the current study warranted the use of absolute values rather than relative due to the standard units of muscle CSA.

\section{Conclusions}

The current study displayed moderate-to-large relationships between VL and BF CSA, back squat and COHS strength measures, and SJ power output. In contrast, trivial relationships existed between strength and maximum potentiation, while small negative relationships existed between CSA and maximum potentiation. These findings provide further information regarding the relationships between muscle CSA, strength, power, and potentiation. It appears that VL and BF CSA may positively influence back squat and COHS strength. Thus, practitioners may consider implementing resistance training methods that improve VL and BF size in order to benefit back squat and COHS performance. Back squat and COHS strength may have a positive influence on SJ power output indicating that squatting variations, both full and partial, may benefit jumping performance. Our results also indicate that muscle CSA and absolute muscular strength may not have much of an influence on maximum SJ PP potentiation; however, it should be noted that relative strength may have a greater influence on maximum potentiation compared to absolute strength.

Acknowledgments: No financial support was provided for the completion of this project or for covering the costs to publish in open access.

Author Contributions: T.J.S. and M.H.S. conceived and designed the experiments; T.J.S. performed the experiments; T.J.S. analyzed the data; M.H.S. contributed materials and analysis tools; T.J.S. and M.H.S. wrote the paper.

Conflicts of Interest: The authors declare no conflict of interest.

\section{References}

1. Minetti, A.E. On the mechanical power of joint extensions as affected by the change in muscle force (or cross-sectional area), ceteris paribus. Eur. J. Appl. Physiol. 2002, 86, 363-369. [CrossRef] [PubMed]

2. Zamparo, P.; Minetti, A.; di Prampero, P. Interplay among the changes of muscle strength, cross-sectional area and maximal explosive power: Theory and facts. Eur. J. Appl. Physiol. 2002, 88, 193-202. [PubMed]

3. DeWeese, B.H.; Hornsby, G.; Stone, M.; Stone, M.H. The training process: Planning for strength-power training in track and field. Part 1: Theoretical aspects. J. Sport Health Sci. 2015, 4, 308-317. [CrossRef] 
4. Suchomel, T.J.; Nimphius, S.; Stone, M.H. The importance of muscular strength in athletic performance. Sports Med. 2016, 46, 1419-1449. [CrossRef] [PubMed]

5. Cormie, P.; McGuigan, M.R.; Newton, R.U. Developing maximal neuromuscular power: Part 2-Training considerations for improving maximal power production. Sports Med. 2011, 41, 125-146. [CrossRef] [PubMed]

6. Suchomel, T.J.; Lamont, H.S.; Moir, G.L. Understanding vertical jump potentiation: A deterministic model. Sports Med. 2016, 46, 809-828. [CrossRef] [PubMed]

7. Seitz, L.B.; Haff, G.G. Factors modulating post-activation potentiation of jump, sprint, throw, and upper-body ballistic performances: A systematic review with meta-analysis. Sports Med. 2016, 46, 231-240. [CrossRef] [PubMed]

8. Tillin, N.A.; Bishop, D. Factors modulating post-activation potentiation and its effect on performance of subsequent explosive activities. Sports Med. 2009, 39, 147-166. [CrossRef] [PubMed]

9. Weeks, B.K.; Gerrits, T.; Horan, S.A.; Beck, B.R. Muscle size not density predicts variance in muscle strength and neuromuscular performance in healthy adult men and women. J. Strength Cond. Res. 2016, 30, 1577-1584. [CrossRef] [PubMed]

10. Gorsuch, J.; Long, J.; Miller, K.; Primeau, K.; Rutledge, S.; Sossong, A.; Durocher, J.J. The effect of squat depth on multiarticular muscle activation in collegiate cross-country runners. J. Strength Cond. Res. 2013, 27, 2619-2625. [CrossRef] [PubMed]

11. Caterisano, A.; Moss, R.E.; Pellinger, T.K.; Woodruff, K.; Lewis, V.C.; Booth, W.; Khadra, T. The effect of back squat depth on the emg activity of 4 superficial hip and thigh muscles. J. Strength Cond. Res. 2002, 16, 428-432. [PubMed]

12. Pereira, G.R.; Leporace, G.; das Virgens Chagas, D.; Furtado, L.F.L.; Praxedes, J.; Batista, L.A. Influence of hip external rotation on hip adductor and rectus femoris myoelectric activity during a dynamic parallel squat. J. Strength Cond. Res. 2010, 24, 2749-2754. [CrossRef] [PubMed]

13. Bryanton, M.A.; Kennedy, M.D.; Carey, J.P.; Chiu, L.Z.F. Effect of squat depth and barbell load on relative muscular effort in squatting. J. Strength Cond. Res. 2012, 26, 2820-2828. [CrossRef] [PubMed]

14. Bellar, D.; Judge, L.W.; Turk, M.; Judge, M. Efficacy of potentiation of performance through overweight implement throws on male and female collegiate and elite weight throwers. J. Strength Cond. Res. 2012, 26, 1469-1474. [CrossRef] [PubMed]

15. Duthie, G.M.; Young, W.B.; Aitken, D.A. The acute effects of heavy loads on jump squat performance: An evaluation of the complex and contrast methods of power development. J. Strength Cond. Res. 2002, 16, 530-538. [CrossRef] [PubMed]

16. Judge, L.W.; Bellar, D.; Craig, B.; Gilreath, E.; Cappos, S.; Thrasher, A. Influence of post activation potentiation on shot put performance of collegiate throwers. J. Strength Cond. Res. 2016, 30, 438-445. [CrossRef] [PubMed]

17. Suchomel, T.J.; Sato, K.; DeWeese, B.H.; Ebben, W.P.; Stone, M.H. Potentiation following ballistic and non-ballistic complexes: The effect of strength level. J. Strength Cond. Res. 2016, 30, 1825-1833. [CrossRef] [PubMed]

18. Seitz, L.B.; de Villarreal, E.S.S.; Haff, G.G. The temporal profile of postactivation potentiation is related to strength level. J. Strength Cond. Res. 2014, 28, 706-715. [CrossRef] [PubMed]

19. Jo, E.; Judelson, D.A.; Brown, L.E.; Coburn, J.W.; Dabbs, N.C. Influence of recovery duration after a potentiating stimulus on muscular power in recreationally trained individuals. J. Strength Cond. Res. 2010, 24, 343-347. [CrossRef] [PubMed]

20. Miyamoto, N.; Wakahara, T.; Ema, R.; Kawakami, Y. Further potentiation of dynamic muscle strength after resistance training. Med. Sci. Sports Exerc. 2013, 45, 1323-1330. [CrossRef] [PubMed]

21. Seitz, L.B.; Trajano, G.S.; Haff, G.G.; Dumke, C.C.L.S.; Tufano, J.J.; Blazevich, A.J. Relationships between maximal strength, muscle size, and myosin heavy chain isoform composition and postactivation potentiation. Appl. Physiol. Nutr. Metab. 2016, 41, 491-497. [CrossRef] [PubMed]

22. Suchomel, T.J.; Sato, K.; DeWeese, B.H.; Ebben, W.P.; Stone, M.H. Relationships between potentiation effects following ballistic half-squats and bilateral symmetry. Int. J. Sports Physiol. Perform. 2015, 11, 448-454. [CrossRef] [PubMed]

23. Suchomel, T.J.; Sato, K.; DeWeese, B.H.; Ebben, W.P.; Stone, M.H. Potentiation effects of half-squats performed in a ballistic or non-ballistic manner. J. Strength Cond. Res. 2016, 30, 1652-1660. [CrossRef] [PubMed] 
24. Reardon, D.; Hoffman, J.R.; Mangine, G.T.; Gonzalez, A.M.; Wells, A.J.; Fukuda, D.H.; Fragala, M.S.; Stout, J.R. Do acute changes in muscle architecture affect post-activation potentiation? J. Sports Sci. Med. 2014, 13, 483-492. [CrossRef] [PubMed]

25. Hopkins, W.G. A Scale of Magnitude for Effect Statistics. Available online: http://sportsci.org/resource/ stats/effectmag.html (accessed on 7 August 2014).

26. Stone, M.H.; O’Bryant, H.; Garhammer, J.; McMillan, J.; Rozenek, R. A theoretical model of strength training. Strength Cond. J. 1982, 4, 36-39. [CrossRef]

27. Kawakami, Y.; Abe, T.; Fukunaga, T. Muscle-fiber pennation angles are greater in hypertrophied than in normal muscles. J. Appl. Physiol. 1993, 74, 2740-2744. [PubMed]

28. Campos, G.E.; Luecke, T.J.; Wendeln, H.K.; Toma, K.; Hagerman, F.C.; Murray, T.F.; Ragg, K.E.; Ratamess, N.A.; Kraemer, W.J.; Staron, R.S. Muscular adaptations in response to three different resistance-training regimens: Specificity of repetition maximum training zones. Eur. J. Appl. Physiol. 2002, 88, 50-60. [CrossRef] [PubMed]

29. Häkkinen, K.; Komi, P.V.; Tesch, P.A. Effect of combined concentric and eccentric strength training and detraining on force-time, muscle fiber and metabolic characteristics of leg extensor muscles. Scand. J. Med. Sci. Sports 1981, 3, 50-58.

30. Wickiewicz, T.L.; Roy, R.R.; Powell, P.L.; Edgerton, V.R. Muscle architecture of the human lower limb. Clin. Orthop. Relat. Res. 1983, 179, 275-283. [CrossRef]

(C) 2017 by the authors. Licensee MDPI, Basel, Switzerland. This article is an open access article distributed under the terms and conditions of the Creative Commons Attribution (CC BY) license (http://creativecommons.org/licenses/by/4.0/). 